\title{
Orthopedics and 3D technology in Turkey: A preliminary report
}

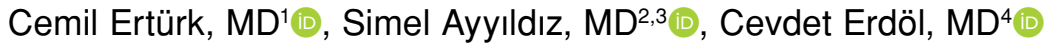 \\ ${ }^{1}$ Department of Orthopaedics and Traumatology, University of Health Sciences, Kanuni Sultan Süleyman Training and Research \\ Hospital, Istanbul, Turkey \\ ${ }^{2}$ Gülhane Medical Design and Manufacturing Application and Research Center, University of Health Sciences Turkey, Ankara, Turkey \\ ${ }^{3}$ Department of Prosthodontics, Gülhane Faculty of Dentistry, University of Health Sciences Turkey, Ankara, Turkey \\ ${ }^{4}$ Rector of University of Health Sciences Turkey, Istanbul, Turkey
}

Three-dimensional (3D) printing technology was invented in the 1980s. In the last decade, 3D printing has a huge impact on the manufacturing industry. ${ }^{[1]}$ With the advancement of medical visualization, the use of $3 \mathrm{D}$ printing materials has become more common in healthcare, education, and research. ${ }^{[2]}$ The 3D printing technology helps us to achieve two major goals in orthopedics surgery: first, the production of 3D printed anatomical models for planning and surgery simulation and, second, the production of custom-made 3D-printed prostheses. ${ }^{[3]}$

For surgeries involving irregular bones such as pelvis or tibial pilon fractures, preoperative planning is particularly difficult in cases of complex anatomy and severe deformity. ${ }^{[4,5]}$ Pilon fractures are usually caused by a high-energy axial load, involved by metaphyseal bone comminution, articular impaction

Received: February 24, 2021

Accepted: April 16, 2021

Published online: June 11, 2021

Correspondence: Cemil Ertürk, MD. SBÜ, Kanuni Sultan Süleyman Eğitim ve Araştırma Hastanesi, Ortopedi ve Travmatoloji Kliniği, 34303 Küçükçekmece, İstanbul, Türkiye.

E-mail: erturkc@yahoo.com

Doi: $10.52312 /$ jdrs.2021.20

Citation: Ertürk C, Ayyıldız S, Erdöl C. Orthopedics and 3D technology in Turkey: A preliminary report. Jt Dis Relat Surg 2021;32(2):279-289.

(02021 All right reserved by the Turkish Joint Diseases Foundation

This is an open access article under the terms of the Creative Commons Attribution-NonCommercial License, which permits use, distribution and reproduction in any medium, provided the original work is properly cited and is not used for commercial purposes (http://creativecommons.org/licenses/by-nc/4.0/).

\section{ABSTRACT}

Objectives: In this study, we present the use of case specific threedimensional (3D) printed plastic models and custom-made acetabular implants in orthopedic surgery.

Materials and methods: Between March 2018 and September 2020, surgeries were simulated using plastic models manufactured by $3 \mathrm{D}$ printers on the two patients with pilon fractures. Also, custom-made acetabular implants were used on two patients with an acetabular bone defect for the revision of total hip arthroplasty (THA).

Results: More comfortable surgeries were experienced in pilon fractures using preoperative plastic models. Similarly, during the follow-up period, the patients that applied custom-made acetabular implants showed a fixed and well-positioning in radiographic examination. These patients did not experience any surgical complications and achieved an excellent recovery.

Conclusion: Preoperative surgical simulation with 3D printed models can increase the comfort of fracture surgeries. Also, custom-made 3D printed acetabular implants can perform an important task in patients treated with revision THA surgery due to severe acetabular defects.

Keywords: Computer-assisted, pilon fracture, revision total hip arthroplasty, three-dimensional, 3D modeling, 3D printing.

and comminution, and severe soft tissue injuries. The main goal of the surgical treatment is the anatomic reduction of the articular surface, restoring length, and reattachment between the metaphysis and diaphysis of bones. ${ }^{[6]}$ These plastic bone models can facilitate a better understanding of the pathoanatomy, and surgeons can use them to simulate surgery. ${ }^{[2,3]}$ In recent years, the use of 3D printed models has enabled the normal 3D anatomy of the tibial plafond on the fracture site to be evaluated and also helped to determine intraoperative contours of the plates. ${ }^{[6,7]}$

Currently, custom-made prosthetic implants that exactly match with patient's bone pathology can be produced with 3D printing technologies. 
The management of massive acetabular bone defects remains a challenging problem, particularly in the revision of total hip arthroplasty (THA) ${ }^{[8,9]}$ Custommade triflange monoblock acetabular components can provide individualized treatment based on the presence of a severe acetabular bone defect.
Such implants can cover a pelvic discontinuity and reconstruct the anatomical hip center. ${ }^{[8-11]}$ However, there is no meta-analysis or randomized-controlled study on 3D print assisted revision hip arthroplasty surgery in the literature. Only case series are reported on this topic.

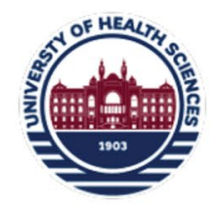

\section{University of Health Sciences Turkey}

(a)

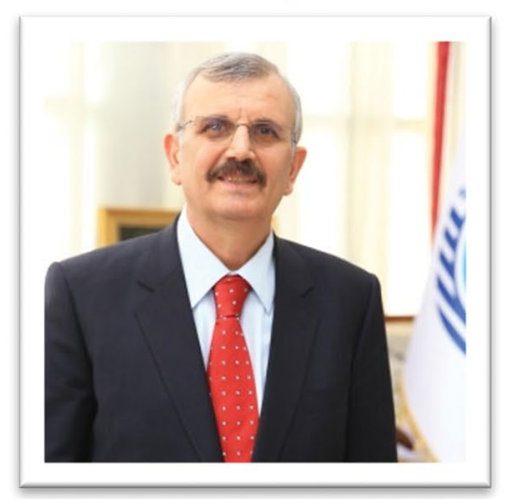

(b)
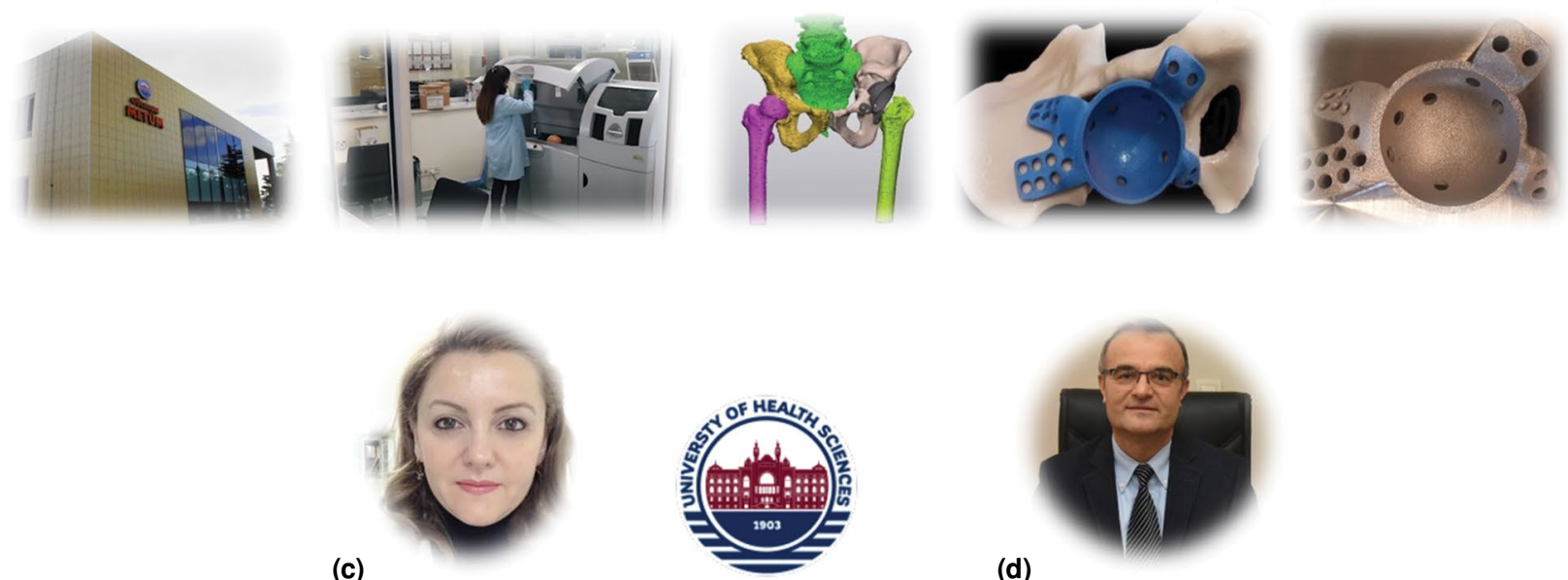

FIGURE 1. (a) Professor Cevdet Erdöl; Rector of Republic of Turkey University of Health Sciences, Istanbul, Turkey. (b) Gülhane Medical Design and Manufacturing Center (GMDMC); using 3D printing technology for production of the custom-made plastic model and acetabular implant. GMDMC is the first and still the only public institution where design and manufacturing are meet under the same roof. (c) Professor Simel Ayyıldız; Director of Gülhane MDMC, Department of Prosthodontics, Gülhane Faculty of Dentistry, University of Health Sciences Turkey, Ankara, Turkey. (d) Professor Cemil Ertürk; Chairperson of Department of Orthopedics and Traumatology, University of Health Sciences, Kanuni Sultan Süleyman Training and Research Hospital, Istanbul, Turkey. 
The University of Health Sciences of Turkey is a specialized university with a health-theme that has 10 medical faculties both in different cities and abroad and has affiliation agreements with more than 60 training and research hospitals across the country. At the same time, there are more than 20 application and research centers with different content within the institutional internal structure. ${ }^{[12]}$ Gülhane Medical Design and Manufacturing Application and Research Center (GMDMC) is one of these centers that was established in Ankara in 2011. The main goal of the center is to design and manufacture custom-made medical implants or prepare presurgical diagnostic or study models for unusual and specific cases. With its know-how, design, and manufacturing capability, GMDMC is an important center in Europe and the world. Also, it is supported by the university and the state (Figure 1). ${ }^{[13]}$ In recent years, the center has produced custom-made medical implants, particularly for veterans, tumor patients, and patients with hard tissue losses in difficult to shape areas. Thus, the service network of the center covers almost all surgical branches. Recently, many customized titanium cranial, sternocostal, pelvic, acetabular, and craniomaxillofacial reconstruction implants and plastic models for surgical preparations have been designed and manufactured in this center. ${ }^{[14]}$

In the present study, four cases were reported to illustrate the surgical experience with implementation of 3D printing at orthopedic surgery. The objectives of the study were (i) to describe the workflow of plastic anatomic models in two patients with pilon fractures and (ii) to evaluate the follow-up results of custom-made monoblock acetabular components in two patients with massive acetabular bone defects.

\section{MATERIALS AND METHODS}

\section{Patients}

Between March 2018 and September 2020, surgeries were simulated using plastic models manufactured by $3 \mathrm{D}$ printers on the two patients with pilon fractures. Also, custom-made acetabular implants were applied to the two patients with acetabular bone defects. A written informed consent was obtained from each patient. The study protocol was approved by the University of Health Sciences, Istanbul Kanuni Sultan Süleyman Training and Research Hospital Ethics Committee (Date/no: 12.02.2021/KAEK-2021.02.45). The study was conducted in accordance with the principles of the Declaration of Helsinki.

\section{Printing the 3D model}

All the models were produced in GMDMC. The 3D bone geometry of patient's extremity reconstructed by medical image processing software $\left(\right.$ Mimics $^{\circledR}$ 19.0, Mimics Innovation Suite, Materialise Medical, Leuven, Belgium). For this purpose, three steps were followed respectively as (i) data acquisition, (ii) image processing of computed tomography (CT) data, and (iii) 3D computer aided design (CAD) modeling of the extremity.

Firstly, CT scans of patients were obtained (Siemens Somatom Emotion 16 CT Scanner; Siemens Healthineers AG, Erlangen, Germany) in Istanbul, Kanuni Sultan Süleyman Training and Research Hospital, University of Health Sciences. The CT data were provided in the Digital Imaging in Medicine and Communications (DICOM) format that is used for medical imaging in thin slices $(512 \times 512$ pixels, pixel size: $0.6-19.2 \mathrm{~mm}$ and slice thickness: $0.75-1.0 \mathrm{~mm}$ ). Then, the CT images were introduced into the Mimics ${ }^{\circledR}$ software to reconstruct the geometrical bony structure of the extremity. The CT scans were processed by the Mimics ${ }^{\circledR}$ software as masking, segmentation, 3D model formation, and reconstruction of the patient's bone model. Finally, the exact 1:1 plastic bone models of the extremity and the mirrored contralateral extremity were

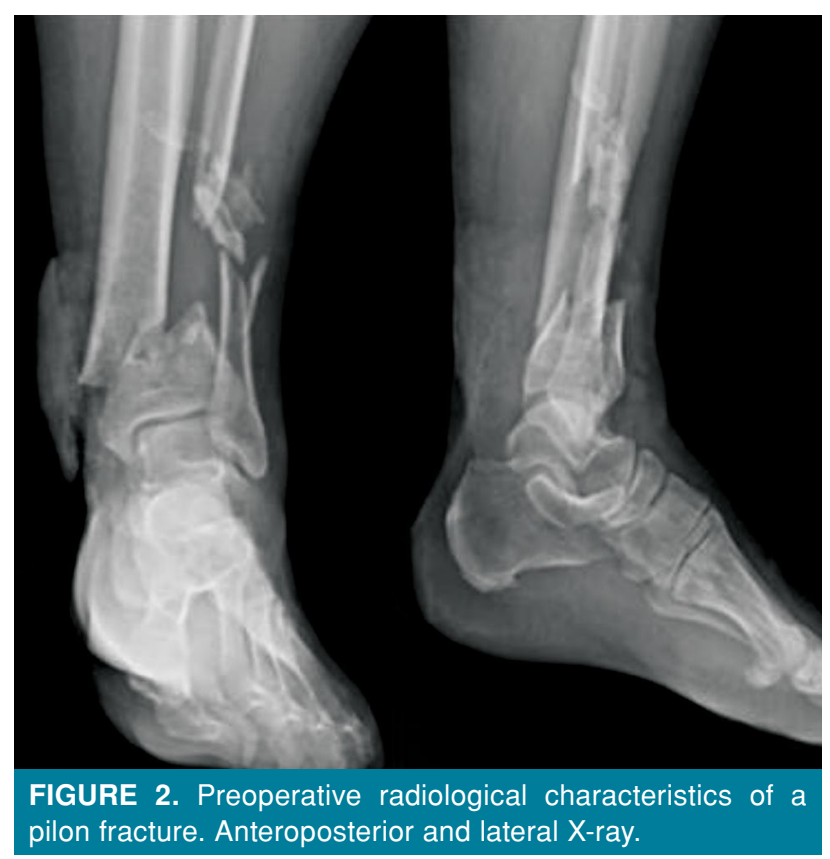



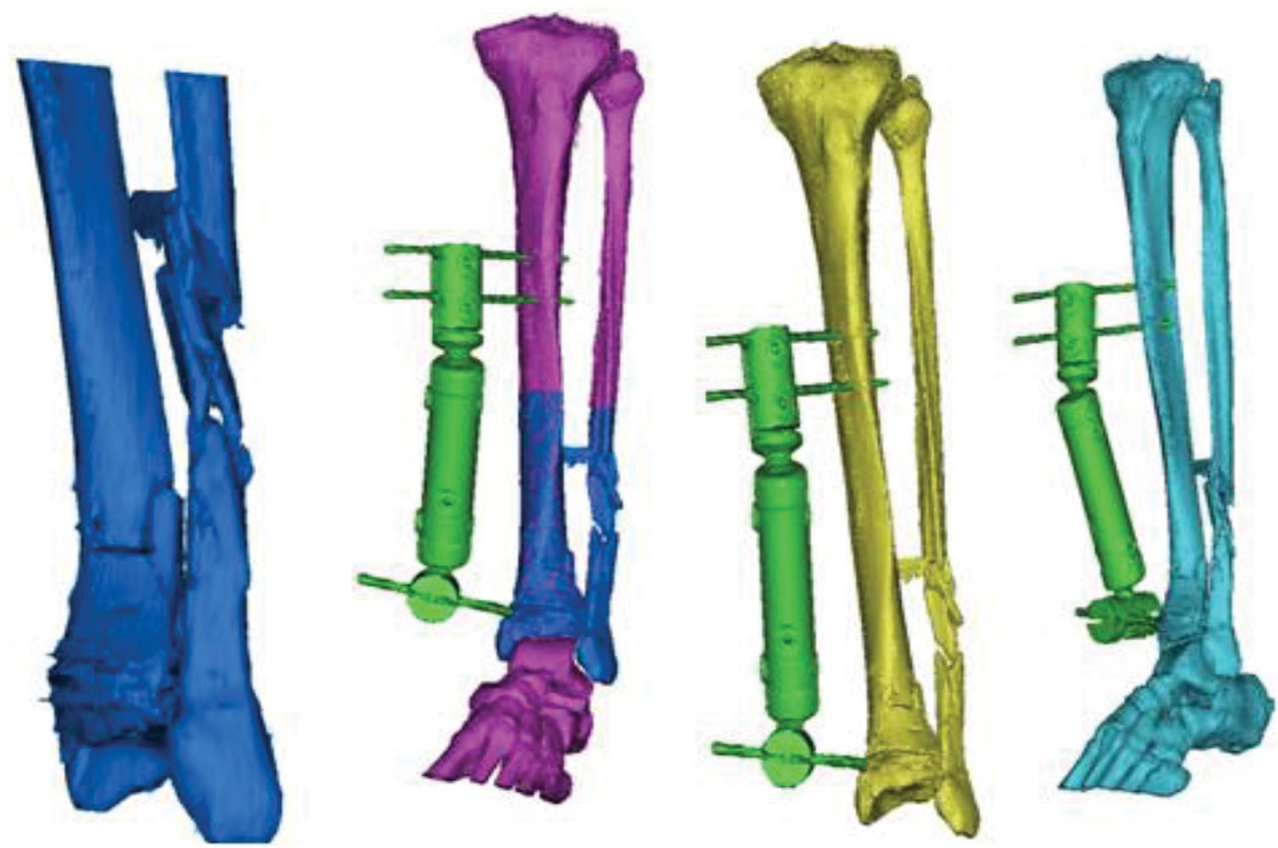

FIGURE 3. The 3D reconstruction and manipulation of the fracture with Mimics ${ }^{\circledR}$ software.

obtained. Plastic models were produced by plastic manufacturing machines using either material jetting (J750, Stratasys, USA) or binder jetting technology (Z Printer 650 Zcorp. USA).

\section{Surgeries}

Patient 1- A 57-year-old man presented to the clinic with a motor vehicle accident history. $\mathrm{He}$ had swelling, abrasions, and ecchymosis on his

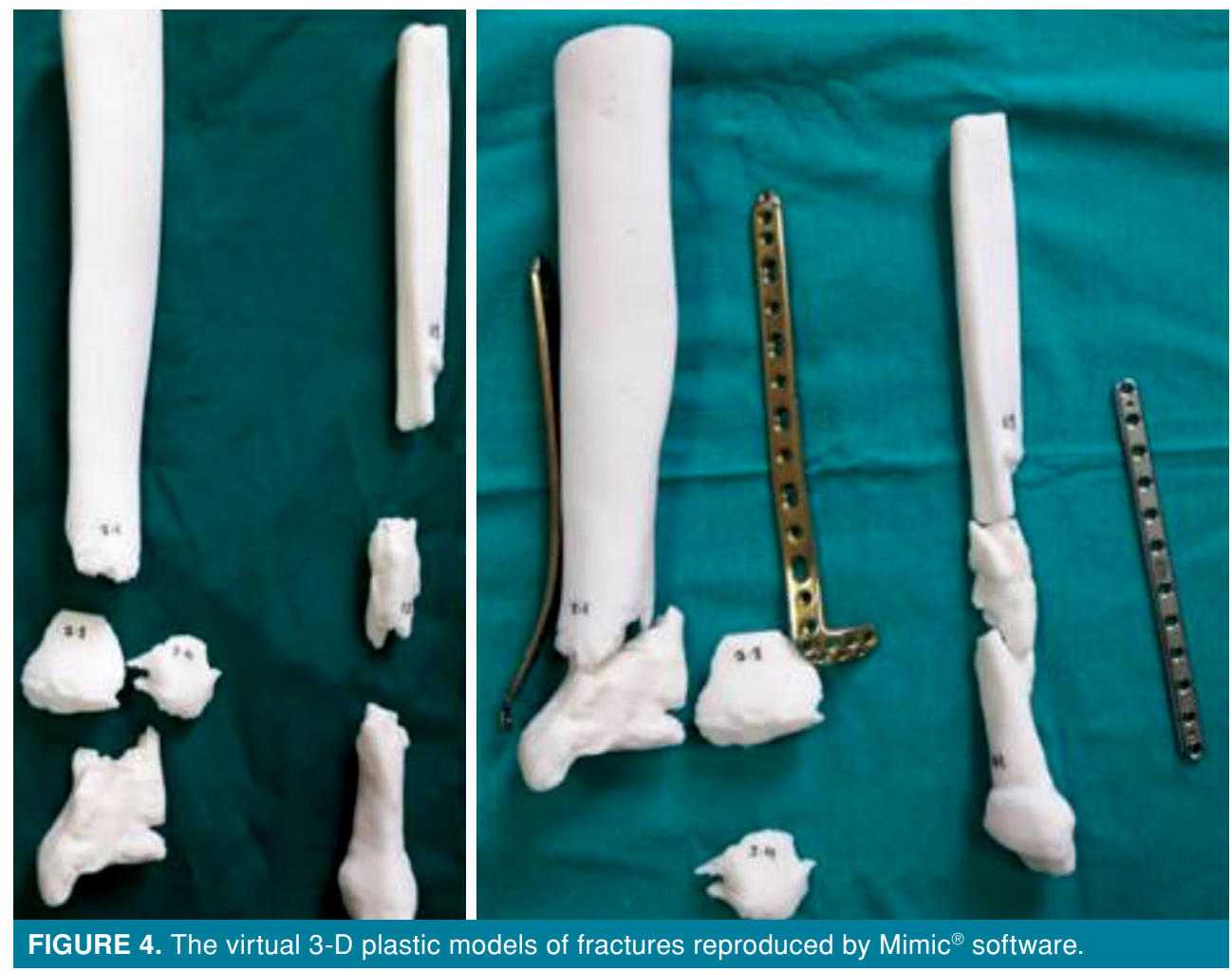




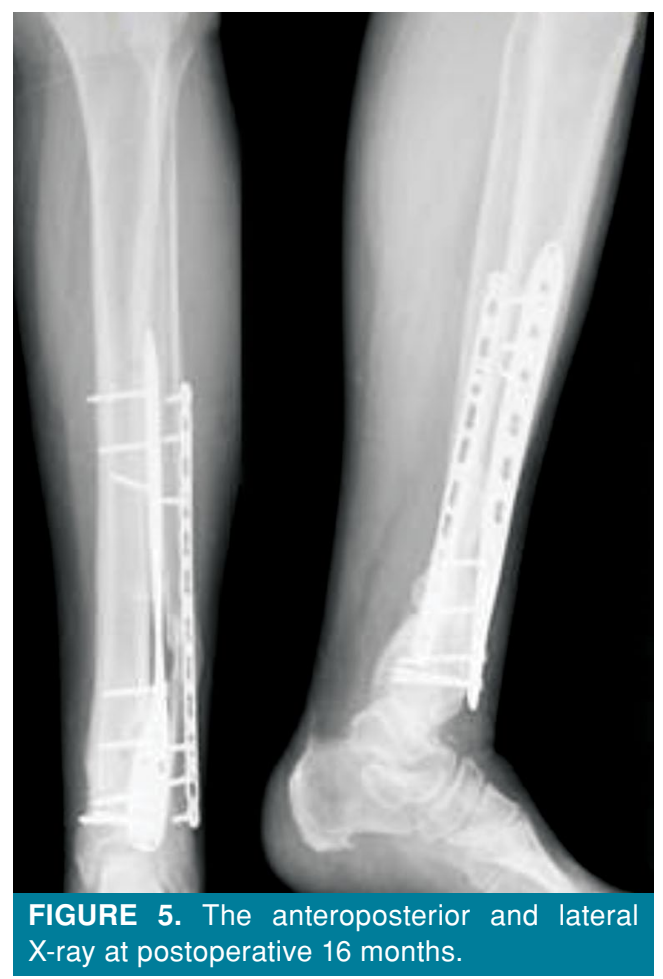

ankle. X-ray and CT scans showed an articular and metaphyseal involvement of tibial plafond and fibula (Figure 2). Two-stage surgery was performed: the initial closed reduction of the fracture stabilization with external fixation. Definitive management such as open reduction and internal fixation was performed, once the soft tissue swelling improved (usually within 10 to 14 days). During this time, CT images in DICOM format were introduced to the
Mimics ${ }^{\circledR}$ software to reconstruct the 3D geometrical bony structure of the ankle (Figure 3). Later, 3D plastic models of the ankle were manufactured (J750, Stratasys, USA).

\section{Surgical simulation}

Before surgery, intraoperative fixation and reduction maneuver imitated accurately on the models. The dimension of the implant could be selected by attaching the plate to the real-size bone model (Figure 4). Furthermore, the plate could be bent to fit the model at the appropriate position with ideal length, location, and orientation. Then, the selected titanium plate and screws were sterilized for surgery.

\section{Surgical methods}

After removal of external fixation, anatomical reduction and stabilization of the articular surface were planned. Then, reduction of the metaphyseal comminution occurred after the joint surface was re-established. Finally, the fracture was fixed by the chosen plate and screws (Figure 5). Pilon fracture surgery was easily performed with the models used before surgery. During the 16-month follow-up, the patient did not experience any surgical complications and he achieved an excellent recovery.

Patient 2- A 25-year-old man who fell from a height was diagnosed with a pilon fracture. Similar procedures were performed in this patient as in Patient 1. The exact (1:1) prototype model of the fracture was produced with 3D printing technology (J750, Stratasys, USA). Simulation of the surgical technique to be used was firstly performed on the plastic model that was produced in 1:1 dimensions

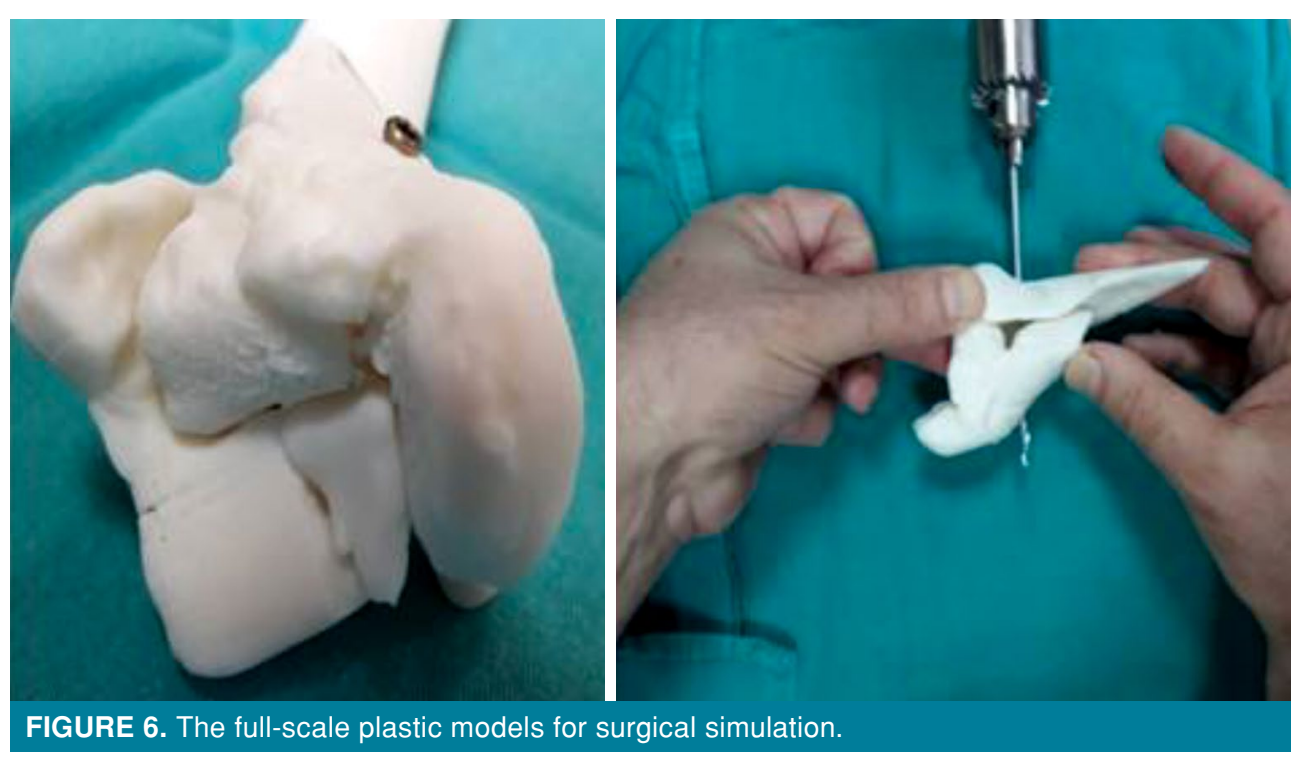




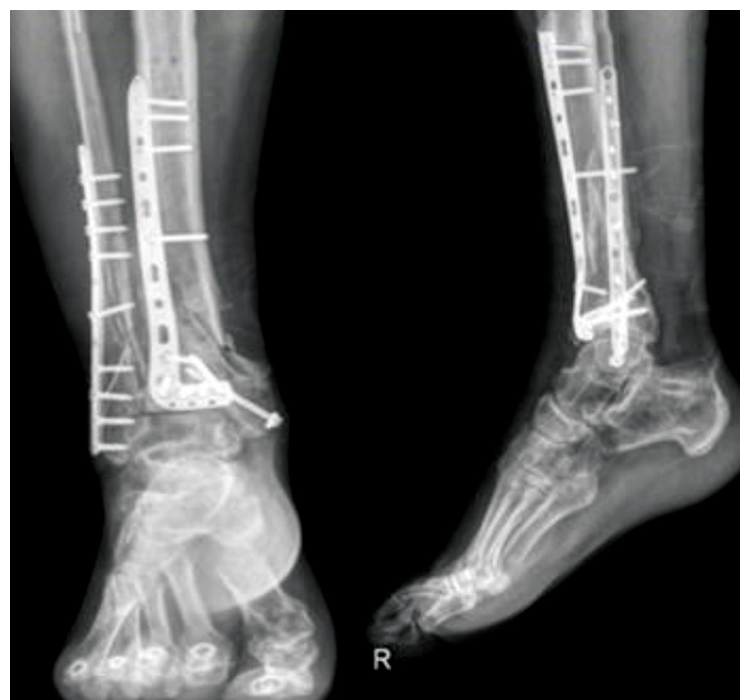

FIGURE 7. The anteroposterior and lateral X-ray at postoperative 14 months. of the fractured area. The result of the simulative operation is shown in Figure 6. The simulative operation is, then, used to guide the actual operation. Postoperative radiographic examination showed satisfactory fracture reduction and fixation. The plate and screw were in a good position (Figure 7). This patient, with a follow-up of 14 months, did not experience any surgical complications and achieved an excellent ankle function.

Patient 3- A 65-year-old female patient presented with worsening right groin and lateral hip pain for six months that was being mobilized in a wheelchair. She had a history of right hip severe coxarthrosis treated with a cementless THA in 2015. Revision surgery was performed in 2016 due to medial protrusion of the acetabular medial wall. The acetabular deficiency was treated by a conventional acetabular component (reinforcement ring). After
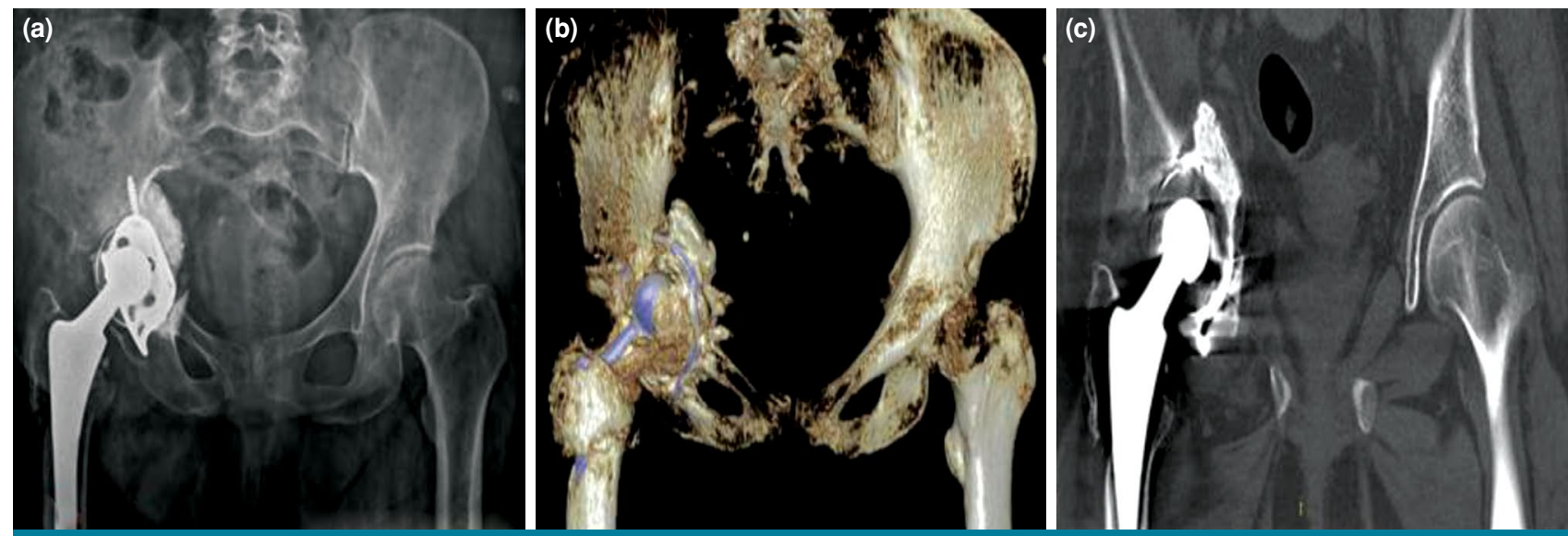

FIGURE 8. Preoperative X-ray (a) and CT scan $(\mathbf{b}, \mathbf{c})$ images showing fabricated acetabular reinforcement ring failure.

(a)

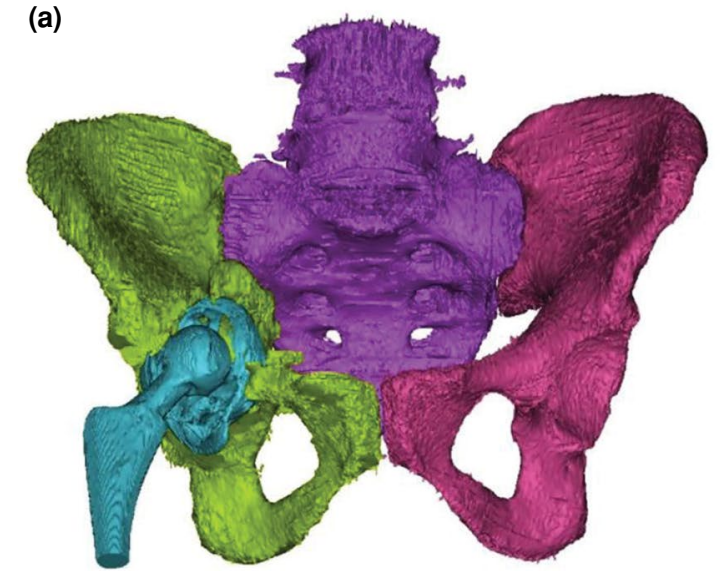

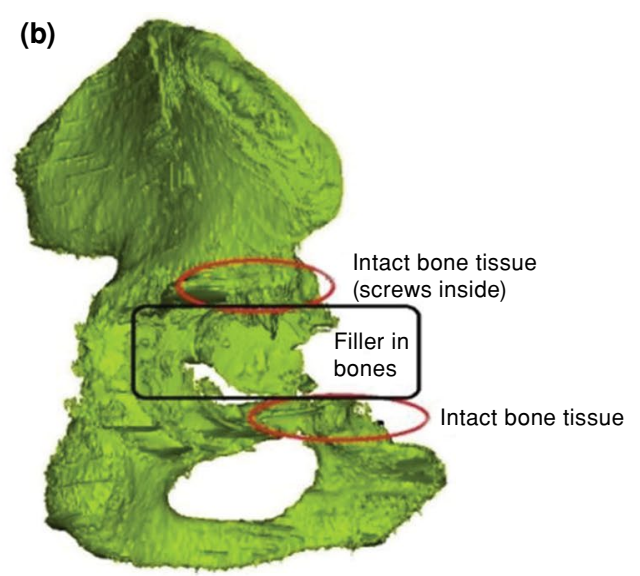

FIGURE 9. 3D images elaborated with the Mimic ${ }^{\circledR}$ software. (a) Entire pelvis with the acetabular ring retained. (b) The acetabular ring was removed during segmentation. 


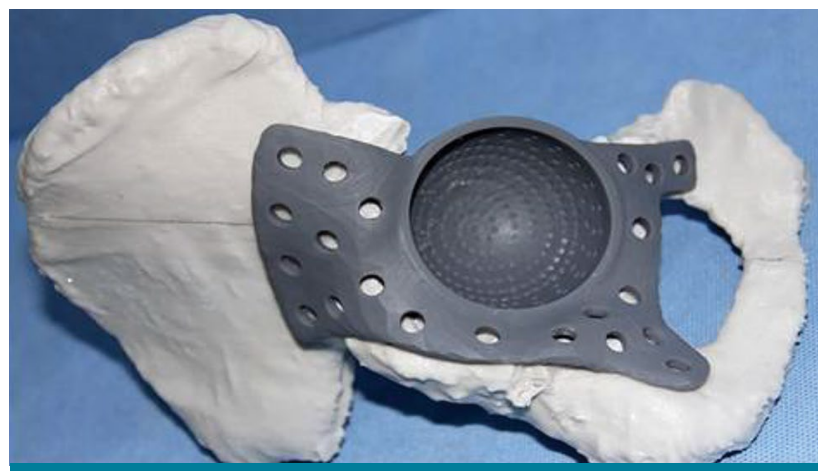

FIGURE 10. 3D production of a plastic acetabular implant and template of the right hemipelvis.

two years, acetabular insufficiency developed again. Firstly, the pelvis of the patient was examined with an anteroposterior (AP) X-ray and, then, a CT scan was taken. A specific metal artefact reduction protocol was used to reduce noise and improve the image quality. The 3D CT scan revealed a failed uncemented THA with Paprosky 3B defect (Figure 8). The DICOM images were transformed into a 3D geometrical bony structure of the hip by Mimics ${ }^{\circledR}$ software (Figures 9a, b).

Firstly, for preoperative planning, the right hip plastic acetabulum model was printed by a 3D printer (Z Printer 650 Zcorp. USA) using high-performance composite powder material that binds with a special liquid binding material. Then, a triflange acetabular implant was designed (3 Matic, Materials, Leuven, Belgium) with a multidisciplinary working between surgeon and engineer. Before the manufacturing of the titanium implant as a final product, a plastic

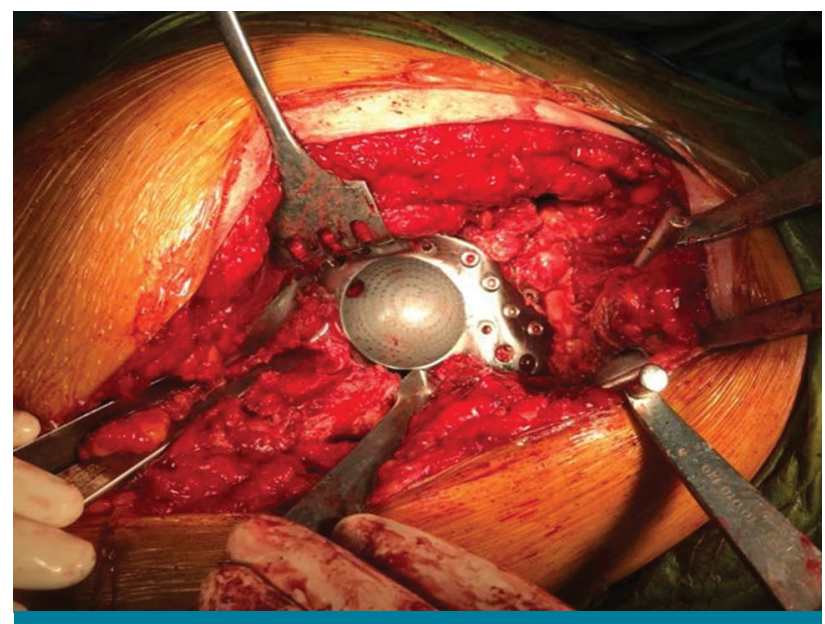

FIGURE 11. An intraoperative image of final implant stabilized with screws. model of the custom-designed medical implant was printed (J750, Stratasys, USA) (Figure 10). After the necessary arrangements were made on the design, soft data were sent to the metal printer to be printed as the final product. The titanium medical implant was printed in 3D by using DMLS technique (M2, Concept Laser GmbH, GE Additive, Lichtenfels, Germany).

\section{Surgical methods}

Using a posterolateral approach to the hip, previous implant was removed. The patient's anatomical landmarks were identified by using the preoperative anatomical plastic model. The position and depth of the acetabular cavity were determined and prepared accordingly. Acetabular cup position was assessed, and the implant was stabilized with screws (Figure 11). Then, an ultra-high molecularweight polyethylene acetabular liner and a metal head were inserted. After the reduction of the joint, the stability of the implant was checked under the guidance of fluoroscopy. There was a fracture in the shaft of the femur. The fracture was fixed by a titanium plate, screws, and cables. Postoperative radiographs showed the accurate placement of the acetabular implant (Figure 12).

Patient 4- Following a complex history of left THA revisions for aseptic loosening, a 64-year-old female presented with coxarthrosis presented with significant left hip pain, inability to mobilize, Paprosky type 3 acetabular defect and pelvic discontinuity (Figure 13a-d). Similar procedures were performed in this patient as in Patient 3 (Figure 14-16).

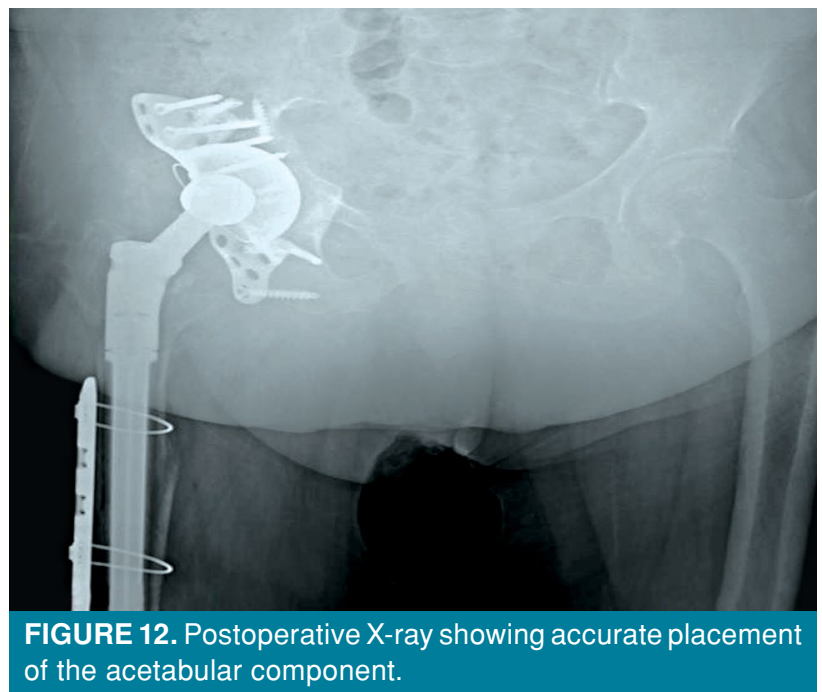



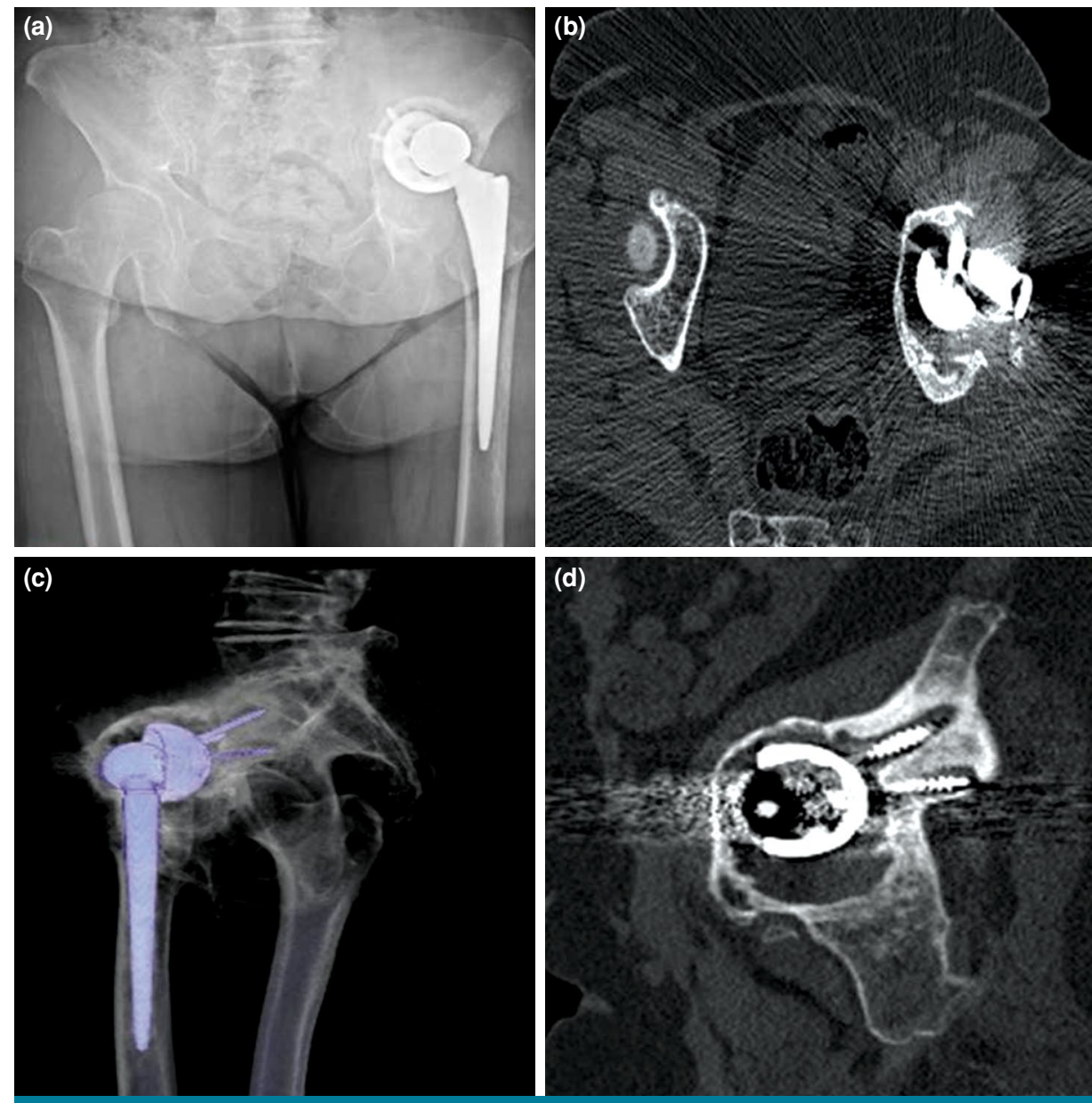

FIGURE 13. Preoperative X-ray (a) and computerized tomography scan (b-d) images showing left acetabular cup failure.
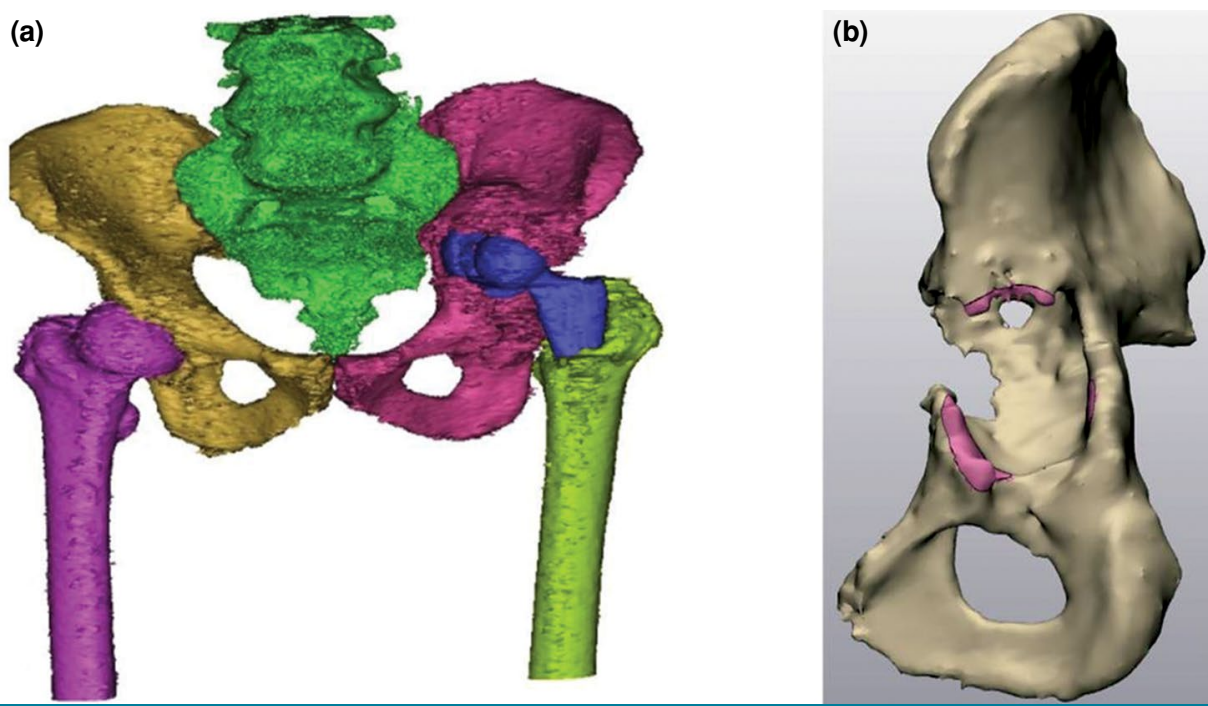

FIGURE 14. 3D images produced with the Mimic $^{\circledR}$ software. (a) Entire pelvis with the acetabular cup and the femoral stem retained. (b) The femoral stem and the acetabular cup were removed during segmentation. 


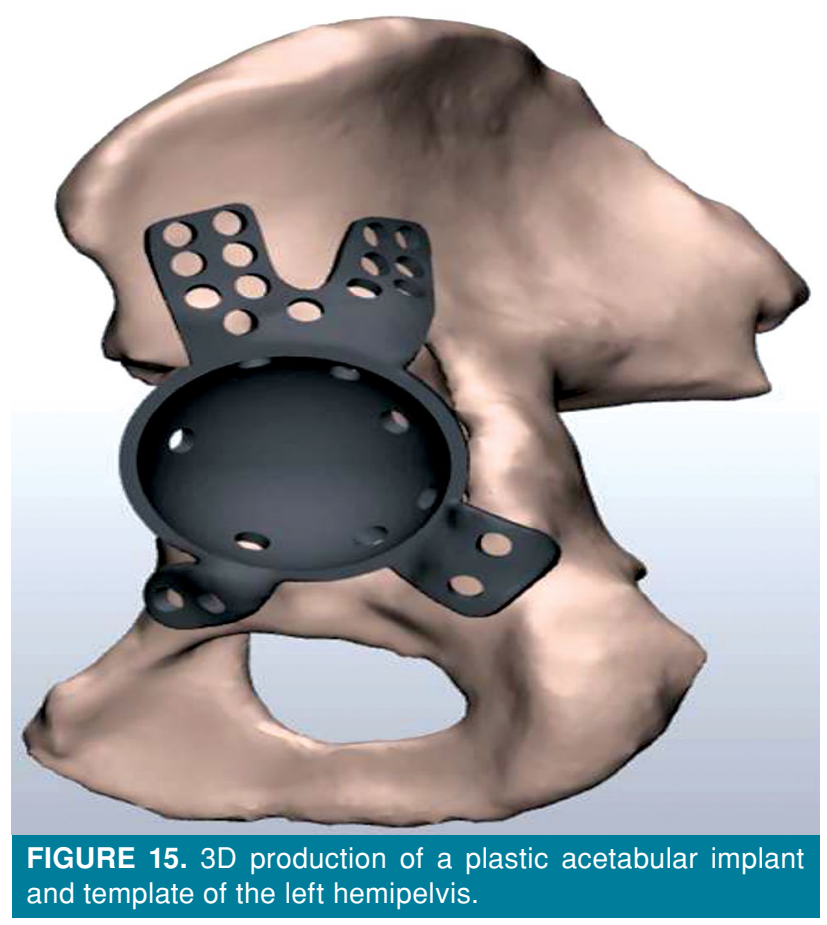

\section{RESULTS}

We performed more comfortably surgery in pilon fractures by using plastic preoperative models. Also, postoperative pelvic radiographs of those patients showed well-positioned and fixed custom-made acetabular implants during follow-up. The patients did not experience any surgical complications and achieved an excellent recovery.

\section{DISCUSSION}

In this study, operations were simulated first on plastic models that produced with 3D printing technology in two trauma patients. Also, it was experienced that custom-made acetabular implants were successfully applied in two patients with massive acetabular defects who underwent THA surgery failure.

Orthopedic surgery, closely related to biomedical engineering, is one of the first medical fields that use 3D printing technology. Preoperative planning is the most important part of all orthopedic surgeries. Preoperative design of anatomical models can provide a significant increase in understanding the patient's bone anatomy and orthopedic deformity. In a multi-center study conducted by Bagira and Chaudhary, ${ }^{[15]} 3 \mathrm{D}$ printed models were found to be valuable tools in orthopedic surgeries involving complex pathoanatomy such as pelvic trauma,

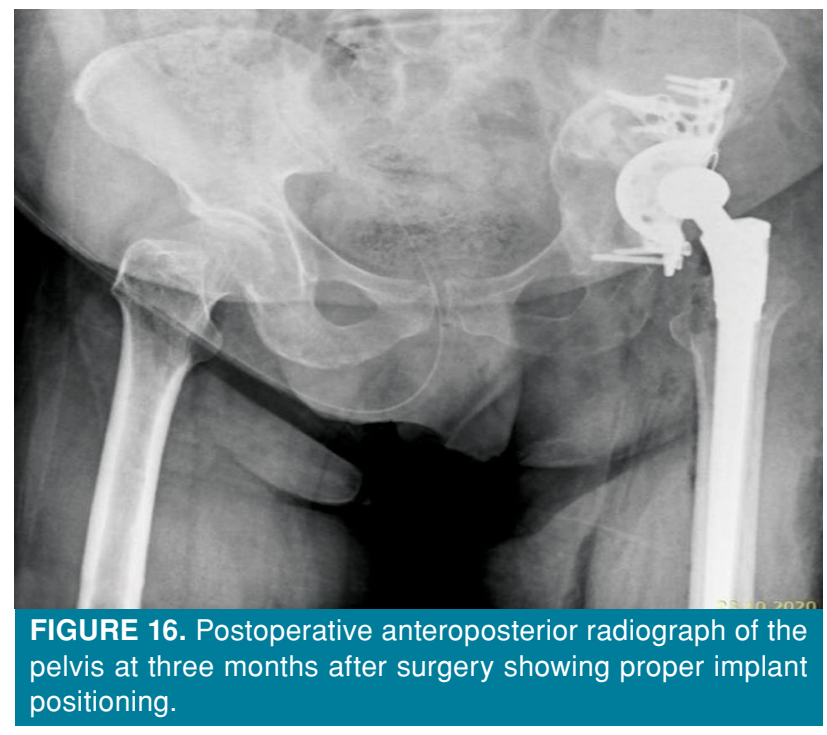

periarticular fracture, and revision arthroplasty. Chen et al. $^{[16]}$ also used a 3D-printed guide template to assist in the achieve accurate placement of plates and screws in the pelvis of 14 adult cadavers. In a similar study, Kang et al. ${ }^{[7]}$ showed that the use of a full-size 3D printed model altered surgeons' choice of preoperative locking plates, particularly when inexperienced surgeons evaluate a complex fracture. Zheng et al. ${ }^{[6]}$ showed that it was possible to use 3D printing technology to treat pilon fractures in clinical practice. In addition, the models produced in the 3D printer have been reported to increase the comfort of surgery for orthopedic treatment with shorter operation time, less blood loss, smaller incision size, and less use of fluoroscopy. ${ }^{[17-19]}$ However, no significant difference has been reported between the groups regarding the rate of infection, fracture union time, traumatic arthritis, and malunion. ${ }^{[19]}$ In our study, surgical procedures that performed for each patient provided a comfortable surgery both for the surgeon and patient, despite the short-term follow-up, similar to the findings of the previous studies. Also, preoperatively selected and bended plates on plastic models were used during surgery without making any change.

Aseptic loosening is the most prevalent cause for THA revision. Acetabular cup revision is a major challenge in the presence of a severe bone defect. Therefore, multiple surgical reconstruction options have been reported for severe acetabular defects such as structural allograft, porous tantalum components, a jumbo acetabular cup, and anti-protrusio cage. ${ }^{[8-10]}$ As the most optimal method for acetabular revision of large defects is 
still unknown, researches are ongoing. Therefore, custom-made 3D printed acetabular implants were developed to achieve primary implant stability, even in cases of pelvic discontinuity situations. ${ }^{[8-10,20]}$ Çıtak et al.$^{[8]}$ reported that 3D-printed acetabular components had a 56\% complication rate in severe acetabular defects, although it was promising in future revision THA. In contrast, Aprato et al. ${ }^{[9]}$ showed encouraging results of the Bespoke acetabular system for Paprosky type 3B acetabular defects and all procedures were rated positively. On the other hand, Kieser et al. ${ }^{[2]}$ reported that the mid-term results of the ossis custom 3D-printed triflange acetabular implant for the management of severe acetabular defects were encouraging. Kavalerskiy et al. ${ }^{[22]}$ presented 17 hip replacement revision surgery patients that were preoperatively planned with 3D models to understand the anatomy and bone defect. However, only three custom-made acetabular implants were used for these patients. In our study, during the follow-up period, no complication was experienced with the custom-made prosthesis that was applied to the patients with Paprosky type 3B acetabular defects. Also, no loosening on the radiographs of the patients were seen and clinical findings gradually improved throughout the follow-up.

Nonetheless, the main limitations of the present study are the limited sample size and relatively short follow-up without a control group. However, this study may be of interest to readers, as it is one of the first applications in our country, that was designed, manufactured, and applied under the same roof of a university.

In conclusion, 3D printers have begun to be used successfully in orthopedics and traumatology, as well as in many fields of medicine. However, its use in orthopedics is relatively new. Besides, its enormous effect and benefit are not well known by most surgeons. In most of the difficult orthopedic cases, preoperative surgical simulation with $3 \mathrm{D}$ printed models can increase the comfort of fracture surgeries. Also, customized 3D printing implants can perform important tasks in joint arthroplasty, when fabricated implants do not fit the size of the patient for severe defects.

\section{Declaration of conflicting interests}

The authors declared no conflicts of interest with respect to the authorship and/or publication of this article.

\section{Funding}

The authors received no financial support for the research and/or authorship of this article.

\section{REFERENCES}

1. Aimar A, Palermo A, Innocenti B. The role of 3D printing in medical applications: A state of the art. J Healthc Eng 2019;2019:5340616.

2. Ye Z, Dun A, Jiang H, Nie C, Zhao S, Wang T, et al. The role of $3 \mathrm{D}$ printed models in the teaching of human anatomy: A systematic review and meta-analysis. BMC Med Educ 2020;20:335.

3. Vaish A, Vaish R. 3D printing and its applications in orthopedics. J Clin Orthop Trauma 2018;9(Suppl 1):S74S75.

4. Saad BN, Yingling JM, Liporace FA, Yoon RS. Pilon fractures: Challenges and solutions. Orthop Res Rev 2019;11:149-57.

5. May H, Köse Ö, Kastan Ö, Emre TY, Sindel M, Akkurt MO. Is there a safe place for posterior malleolar screw fixation? An anatomic study on dry bones. Jt Dis Relat Surg 2020;31:476-9.

6. Zheng W, Chen C, Zhang C, Tao Z, Cai L. The feasibility of $3 \mathrm{D}$ printing technology on the treatment of pilon fracture and its effect on doctor-patient communication. Biomed Res Int 2018;2018:8054698.

7. Kang HJ, Kim BS, Kim SM, Kim YM, Kim HN, Park JY, et al. Can preoperative $3 \mathrm{D}$ printing change surgeon's operative plan for distal tibia fracture? Biomed Res Int 2019;2019:7059413.

8. Citak M, Kochsiek L, Gehrke T, Haasper C, Suero EM, Mau H. Preliminary results of a 3D-printed acetabular component in the management of extensive defects. Hip Int 2018;28:266-71.

9. Aprato A, Giachino M, Bedino P, Mellano D, Piana R, Massè A. Management of Paprosky type three B acetabular defects by custom-made components: Early results. Int Orthop 2019;43:117-22.

10. Trauner KB. The emerging role of $3 \mathrm{D}$ printing in arthroplasty and orthopedics. J Arthroplasty 2018;33:2352-4.

11. Zhang H, Liu Y, Dong Q, Guan J, Zhou J. Novel 3D printed integral customized acetabular prosthesis for anatomical rotation center restoration in hip arthroplasty for developmental dysplasia of the hip crowe type III: A case report. Medicine (Baltimore) 2020;99:e22578.

12. Sağlık Bilimleri Üniversitesi. Available at: https://www. sbu.edu.tr/ [Accessed: November 20, 2018]

13. Available at: https://www.sbu.edu.tr/tr/arastirma/ uygulama-ve-arastirma-merkezleri/gulhane-medikaltasarim-ve-uretim-uam/index [Accessed: December 17, 2019]

14. Available at: http://www.uhs.edu.tr/research/medicaldesign-and-manufacturing-center [Accessed: February 20, 2021]

15. Bagaria V, Chaudhary K. A paradigm shift in surgical planning and simulation using 3D graphy: Experience of first 50 surgeries done using 3D-printed biomodels. Injury 2017;48:2501-8.

16. Chen $X$, Chen $X$, Zhang G, Lin $H, Y u ~ Z, W u ~ C$, et al. Accurate fixation of plates and screws for the treatment of acetabular fractures using 3D-printed guiding templates: An experimental study. Injury 2017;48:1147-54.

17. Zheng W, Su J, Cai L, Lou Y, Wang J, Guo X, et al. Application of 3D-printing technology in the treatment of humeral intercondylar fractures. Orthop Traumatol Surg Res 2018;104:83-8. 
18. Zhang YW, Xiao X, Xiao Y, Chen X, Zhang SL, Deng L. Efficacy and prognosis of $3 \mathrm{D}$ printing technology in treatment of high-energy trans-syndesmotic ankle fracture dislocation "log-splitter" injury. Med Sci Monit 2019;25:4233-43.

19. Bai J, Wang Y, Zhang P, Liu M, Wang P, Wang J, et al. Efficacy and safety of 3D print-assisted surgery for the treatment of pilon fractures: A meta-analysis of randomized controlled trials. J Orthop Surg Res 2018;13:283.

20. Li Q, Chen X, Lin B, Ma Y, Liao JX, Zheng Q. Threedimensional technology assisted trabecular metal cup and augments positioning in revision total hip arthroplasty with complex acetabular defects. J Orthop Surg Res 2019;14:431.

21. Kieser DC, Ailabouni R, Kieser SCJ, Wyatt MC, Armour PC, Coates MH, et al. The use of an Ossis custom 3D-printed tri-flanged acetabular implant for major bone loss: minimum 2-year follow-up. Hip Int 2018;28:668-74.

22. Kavalerskiy GM, Murylev VY, Rukin YA, Elizarov PM, Lychagin AV, Tselisheva EY. Three-dimensional models in planning of revision hip arthroplasty with complex acetabular defects. Indian J Orthop 2018;52:625-30. 